\title{
Review
}

\section{A meta-analysis of prevalence rates and moderating factors for cancer-related post-traumatic stress disorder}

\author{
Gareth Abbey '* Simon B. N. Thompson ', Tamas Hickish ${ }^{2,3}$ and David Heathcote ' \\ 'Faculty of Science \& Technology, Psychology Research Centre, Bournemouth University, Talbot Campus, Poole, UK \\ ${ }^{2}$ The Royal Bournemouth and Christchurch Hospitals NHS Foundation Trust, Bournemouth, Dorset, UK \\ ${ }^{3}$ School of Healthcare Sciences, Psychology Research Centre, Bournemouth University, Talbot Campus, Poole, UK
}

* Correspondence to:

Faculty of Science \& Technology,

Psychology Research Centre, Bournemouth University, Talbot Campus, Poole House-PG 104 Fern Barrow, Poole, BHI 2 5BB, UK. E-mail: garethabbey@ hotmail.com

Received: 2 October 2013

Revised: 23 July 2014

Accepted: 25 July 2014

\begin{abstract}
\end{abstract}
Objective: Systematic reviews highlight a broad range of cancer-related post-traumatic stress disorder (CR-PTSD) prevalence estimates in cancer survivors. This meta-analysis was conducted to provide a prevalence estimate of significant CR-PTSD symptoms and full diagnoses to facilitate the psychological aftercare of cancer survivors.

Methods: A systematic literature search was conducted for studies using samples of cancer survivors by using validated clinical interviews and questionnaires to assess the prevalence of CR-PTSD $(k=25$, $n=4189$ ). Prevalence estimates were calculated for each assessment method using random-effects meta-analysis. Mixed-effects meta-regression and categorical analyses were used to investigate study-level moderator effects.

Results: Studies using the PTSD Checklist-Civilian Version yielded lower event rates using cut-off $[7.3 \%, 95 \%$ confidence intervals $(C I)=4.5-11.7, k=10]$ than $\operatorname{symptom}$ cluster $(11.2 \%, 95 \% \quad C I=8.7-$ $14.4, k=9)$. Studies using the Structured Clinical Interview for Diagnostic and Statistical Manual, Fourth Edition (SCID), yielded low rates for lifetime $(15.3 \%, 95 \% \mathrm{CI}=9.1-25, k=5)$ and current CR-PTSD $(5.1 \%, 95 \% \mathrm{CI}=\mathbf{2 . 8}-\mathbf{8 . 9}, k=9)$. Between-study heterogeneity was substantial $\left(I^{2}=54-87 \%\right)$. Studies with advanced-stage samples yielded significantly higher rates with PTSD Checklist-Civilian Version cluster scoring $(p=0.05)$, and when assessing current CR-PTSD on the SCID $(p=0.05)$. The effect of mean age on current PTSD prevalence met significance on the SCID $(p=0.05)$. SCID lifetime prevalence rates decreased with time post-treatment $\left(R^{2}=0.56, p<0.05\right)$.

Discussion: The cancer experience is sufficiently traumatic to induce PTSD in a minority of cancer survivors. Post-hoc analyses suggest that those who are younger, are diagnosed with more advanced disease and recently completed treatment may be at greater risk of PTSD. More research is needed to investigate vulnerability factors for PTSD in cancer survivors.

(C) 2014 The Authors. Psycho-Oncology published by John Wiley \& Sons Ltd.

\section{Introduction}

Systematic reviews show that long-term cancer survivorship is accompanied by co-morbid depression, anxiety and symptoms of post-traumatic stress disorder (PTSD) [1-4]. The PTSD Field Trials for the Diagnostic and Statistical Manual, Fourth Edition (DSM-IV) [5], revealed that $22 \%$ of cancer survivors present with lifetime cancerrelated PTSD (CR-PTSD) [6], endorsing cancer diagnosis and treatment as a traumatic stressor. This inspired research into the prevalence and presentation of CR-PTSD in cancer populations [7].

According to DSM-IV [5], PTSD can develop when an individual experiences a traumatic event that threatens their psychological and/or physical integrity (criterion A1), and they react with helplessness or horror (criterion A2). They typically re-experience the memories, sensations and emotions from the event through sensory flashbacks and nightmares (criterion B), avoid trauma reminders and can be emotionally numb (criterion $\mathrm{C}$ ), anxious, irritable and hyper-vigilant (criterion $\mathrm{D}$ ). These symptoms are pathological when their duration is more than 1 month post-trauma (criterion E) and causes socio-occupational impairments (criterion F). Using the DSM-IV criteria, studies to date identified lifetime rates from 5\% [7] to 35\% [8]. However, the recent publication of the DSM-5 [9] challenges this position. The new trauma criterion states '.... life-threatening...or debilitating medical condition is not necessarily considered a traumatic event. Medical incidents that qualify as traumatic events involve sudden, catastrophic events [e.g., waking during surgery, anaphylactic shock]' (p. 274).

A recent article by Kangas [10] discussed how the new PTSD diagnostic criteria would affect the psychological aftercare of individuals who have completed cancer treatment-a population we will define as cancer survivors. Consequently, a PTSD diagnosis is less likely to be given to those who present with PTSD symptoms, in favour of DSM-5's adjustment disorder (AD). AD is a diagnosis 
given to individuals who either (a) present with subsyndromal PTSD or (b) experience the complete PTSD syndrome in response to an event that is not considered traumatic [9]. Kangas [10] argued that relatively few DSM-IV studies investigated the rates of $\mathrm{AD}$ and PTSD in cancer samples and, thus, could not determine if PTSD was the primary disorder. This casts doubt on the appropriateness of PTSD as a diagnosis for cancer survivors but emphasises the need to correctly diagnose a cancer survivor who presents with trauma/stress-related symptoms.

It does not, however, address the needs of oncology specialists to know the proportion of patients who would likely develop PTSD or AD, or of the factors contributing to their presentation. In systematic reviews to date [1-4], efforts to achieve a prevalence estimate have been impeded owing to the substantial variability in rates, which is often attributed to extreme between-study heterogeneity in assessment methods (e.g. questionnaire versus clinical interview), assessment points (post-diagnosis or posttreatment) and sample characteristics such as severity of disease, or mean age [11].

Firstly, many studies use different methods of estimating (or diagnosing) PTSD. The most obvious example of this is the use of clinical interviews, such as the 'gold standard' Clinician-Administered PTSD Scale (CAPS) [12], or the Structured Clinical Interview for DSM-IV (SCID) [13], which yields a full DSM-IV PTSD diagnosis (criteria AF), versus screening questionnaires such as the PTSD Checklist - Civilian Version (PCL-C) [14], which assesses symptom severity (criteria B-D). Although the PCL-C has been criticised for not establishing the fulfilment of PTSD criterion A [15], it has been administered to cancer patients and survivors in the context of experiencing cancer as a traumatic event (criterion A1). It can also assess PTSD symptoms over the past month (criterion E) and has been used frequently to assess PTSD symptom severity and provide a tentative PTSD diagnosis. Andrykowski et al. [16] validated the PCL-C against the SCID [13], and its factor structure has been corroborated in cancer survivors $[17,18]$. Therefore, the PCL-C is arguably a useful questionnaire to assess PTSD symptom severity and has sufficient sensitivity and specificity to screen for a probable PTSD diagnosis. Nevertheless, like other screening questionnaires, the PCL-C has been known to inflate PTSD prevalence rates owing to (a) the endorsement of PCL-C items that are confounded by disease symptoms [19]; (b) the inability to assess functional impairment (criterion F); and (c) whether cut-off scores are used or using a symptom cluster method by imitating the DSM-IV-TR PTSD diagnostic criteria [20]. The proximity of psychological assessment (by either questionnaire or structured interview) to the end of diagnosis $o r$ treatment may also contribute to prevalence. Evidence for this is equivocal; some studies have shown that PTSD persists even 20 years after the cancer experience [21], whereas others reveal no relationship between PTSD symptom severity and time since diagnosis [6,22-25] or time since treatment [26,27].

Secondly, heterogeneity may emerge from sample characteristics. Younger age at cancer diagnosis is associated with more severe PTSD symptoms [15,17,23,24,26,28-31], although some studies have not found a significant association [21,25,27]. Recent meta-analyses have demonstrated that younger samples of medical trauma survivors are associated with higher PTSD prevalence rates [32]. The disease characteristics of each study sample may also contribute. Gurevich et al. [1] argued that many studies that report no relationship between disease severity and CRPTSD prevalence rates are actually skewed towards early disease stages $[6,15,26,28-30]$. However, samples that are more evenly distributed report significant effects of disease staging on prevalence rates $[8,27,33,34]$. In order to counter this heterogeneity, it has been argued that future studies of PTSD in cancer populations would benefit from larger, broader samples [35]. Therefore, a meta-analysis of studies reporting CR-PTSD prevalence statistics was conducted in order to find an empirical estimate of the proportion of adult cancer survivors who will present with (a) clinically significant PTSD symptoms or (b) a full diagnosis of PTSD under DSM-IV criteria. Our aims were as follows:

1. To establish a mean PTSD prevalence estimate for CR-PTSD in adult cancer survivors, with regard to the use of diagnostic interview methods and screening questionnaires. This will provide an estimate of the presentation of full-PTSD, and caseness-level PTSD symptoms indicating the need for a full assessment, respectively.

2. To establish what percentage of variance in prevalence estimates can be explained by between-study heterogeneity, with a focus on disease-stage sample characteristics, mean sample age and time since diagnosis or treatment. This will provide evidence for patient variables that may be risk factors for the development of PTSD.

\section{Method}

\section{Search strategy}

Seven online databases (MEDLINE, PsycARTICLES, PsycINFO, CINAHL Complete, CINAHL Plus, Academic Search and E-Journals) were searched systematically. Studies published from 1994 (the publication of the DSM-IV) to 11 June 2013 were included. The search terms (cancer) AND (PTSD) AND (prevalence) were used for every database. It was decided to adopt such broad terms to capture as many studies as possible that are focused on the number of CR-PTSD cases in their sample. Dissertations were included in the search, but case studies, and studies that were unavailable in English, were 
excluded. Systematic reviews from 1999 onwards concerning CR-PTSD in survivors of adult cancers were examined, and any relevant references from these reviews that were not returned in online databases were abstracted. The following inclusion/exclusion criteria were compiled by the primary and secondary authors following an investigation of issues identified in the aforementioned systematic reviews and were agreed on by consensus of all the authors.

\section{Inclusion and exclusion criteria}

Articles were included if they met these criteria: (1) they were conducted with, and specify, a sample of cancer patients (those who are in treatment) or survivors (those who have completed treatment). Note that the search strategy does not specify any particular cancer owing to the systematic reviews arguing the scarcity of evidence for the effect of disease variables on CR-PTSD prevalence rates; (2) they provide PTSD prevalence estimates (i.e. the number of people in the sample who meet caseness); (3) the prevalence statistics are in reference to cancer diagnosis and treatment as the traumatic stressor (CR-PTSD); (4) they provide summary statistics on (a) disease stages in the sample, (b) gender, (c) mean age of sample and (d) mean time post-diagnosis or post-treatment; (5) they use both or either of the structured clinical interviews such as the CAPS [12], SCID [13], Watson's PTSD Inventory [36] or a validated screening questionnaire that is based on DSM-IV PTSD criteria such as the PCL-C [14], or PTSD Reaction Index [37]; and (6) use cross-sectional, or longitudinal/prospective, methods. Articles were excluded if they (1) used the Impact of Events Scale as a standalone measure of probable PTSD [38]; (2) specifically used samples of adult survivors of childhood cancers; or (3) used the same sample as another included study.

\section{Study selection}

After the removal of duplicate entries, the full texts of the remaining records were read by the primary author. Studies that clearly met exclusion criteria (e.g. did not use cancer samples or used the Impact of Events Scale as a standalone PTSD measure) were excluded by the primary author. All authors read the remaining full-text articles and selected from the remaining studies those that they believed met inclusion criteria, and discrepancy between authors was resolved by full consensus.

\section{Data abstraction}

All prevalence figures, sample characteristics and study methods were abstracted onto a spreadsheet for review by the primary author and re-checked four times to ensure accurate data abstraction, and a random sample of the included studies was independently checked by a clinical psychology postgraduate (Table 1). There was full agreement. Although most of the data are easily abstracted in their presented form (e.g. mean age, gender, event rates and assessment method), some statistics were calculated manually. For example, in order to assess the moderating effect of disease-stage distribution on CR-PTSD prevalence rates, a summary statistic on the skew of the sample distribution had to be used. Given that many studies are skewed towards low disease stages, the mode $(0,1,2,3$ or 4$)$ was used to depict the disease stage that was most frequent in each sample. There were $k=4$ studies that were bimodal; in these cases, all bimodal distributions were characterised by I-II, II-III or III-IV, so given the nature of cancer-stage progression, the mean was used $(1.5,2.5$ and 3.5 , respectively). In some cases, the sample size reported in abstracts did not reflect that in the final analysis, so our abstracted $n$ s reflected only those participants who completed the study. Finally, some authors reported time since diagnosis or treatment using different units (days/weeks rather than months). All studies were converted to the number of months. In all cases where it was reported in days or weeks, a month was treated as 30 days, and weeks were multiplied by seven and then divided by 30 to obtain the number of months.

\section{A priori statistical methods and analysis}

All statistical analyses and graphical presentations were conducted using Comprehensive Meta-analysis (CMA) [39]. Prevalence statistics were depicted using the event rate. Ninety-five percent confidence intervals (CIs) were calculated within the CMA software using the sample size (n) and standard error. When prospective studies were included in the analysis, the time 1 (T1) measurements (being temporally associated with study-level moderators such as time since diagnosis and/or treatment) were used to calculate the event rates. In cases where the $\mathrm{T} 1$ measurements were not post-treatment, the $\mathrm{T} 2$ prevalence estimates (and the mean time post-treatment) were used. This was the case for one study only [34]. All metaanalyses were conducted under a random-effects model owing to the substantial methodological heterogeneity identified in systematic reviews. Mixed-effects metaregression was used to examine the association between continuous variables such as time since diagnosis or treatment end and mean age of sample, on CR-PTSD prevalence rates. Categorical analyses were conducted on studies where samples were characterised by either early (I-II) or advanced (III-IV) disease stages, and also on population studies (USA/Canada, UK/Europe and eastern countries). Finally, publication bias was assessed using funnel plots. These plots depict the spread of prevalence rates according to (a) interviews and screeners, (b) symptom cluster and cut-off scoring for screening questionnaires and (c) current versus lifetime CR-PTSD for 
374

G. Abbey et al.

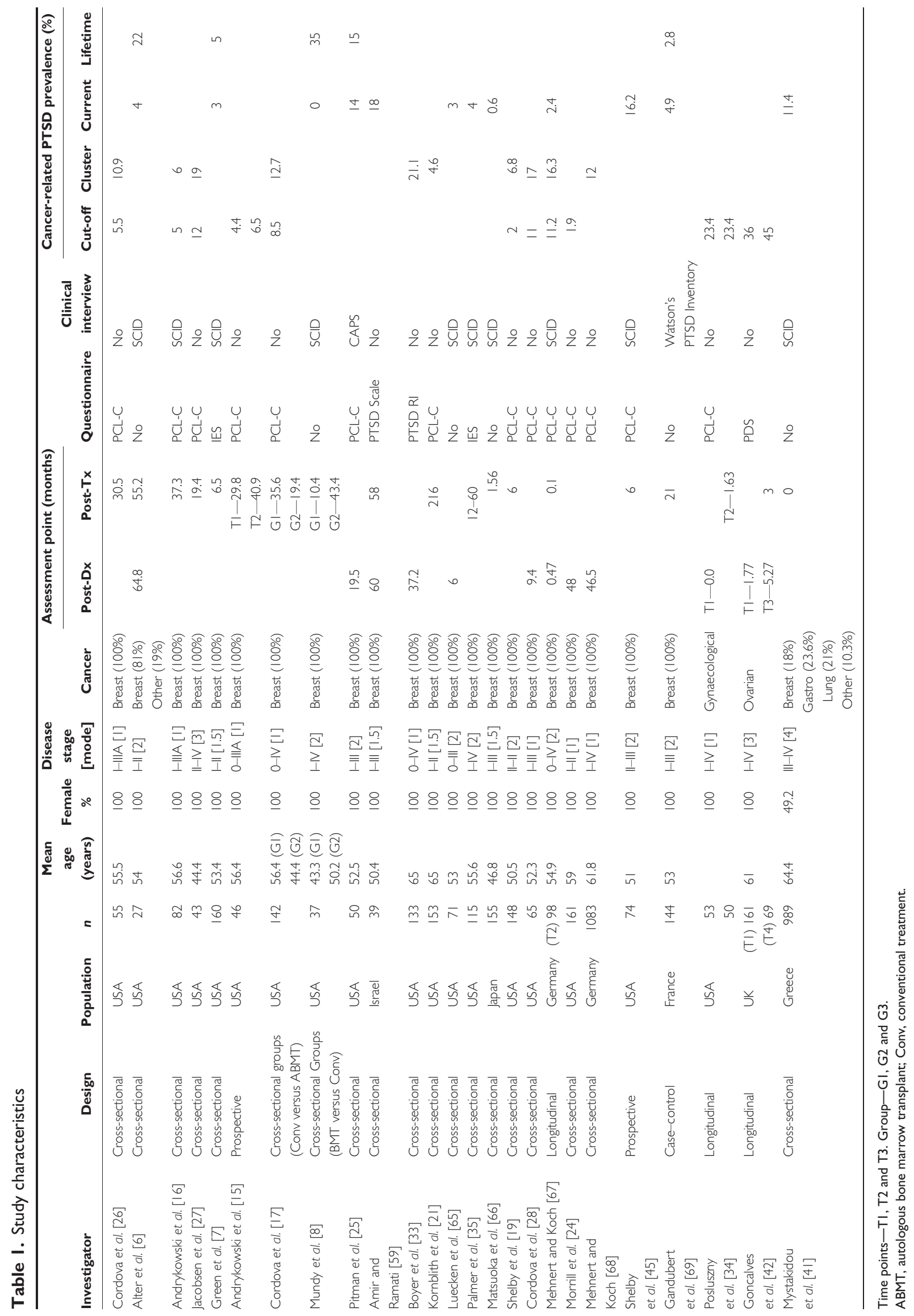

(C) 2014 The Authors. Psycho-Oncology published by John Wiley \& Sons Ltd.

Psycho-Oncology 24: 37I-38I (2015)

DOI: $10.1002 /$ pon 
clinical interviews. In order to calculate the variance contribution of statistically significant study-level moderators on PTSD event rates, we calculated the $R^{2}$ statistic using the following formula: $\left[R^{2}=1-\left(T_{\text {unexplained }}^{2} / T_{\text {total }}^{2}\right)\right]$.

\section{Results}

\section{Literature search and study characteristics}

We identified 257 articles. After duplicates were removed, 109 articles remained. A combined total of 138 studies were recovered from online databases and systematic reviews. Figure 1 depicts the PRISMA search strategy [40]. Table 1 presents the abstracted prevalence data and study/sampling characteristics for the final dataset $(k=25)$. The aggregated sample was $n=4189$, of which $88 \%$ were female, and the study of Mystakidou et al. [41] was the only study to represent both genders. In terms of methodology, $k=19$ studies were cross-sectional, and $k=6$ were longitudinal/prospective. The majority of studies were conducted in the USA $(k=18)$, with one in Japan, five in Europe and the UK, and one in Israel. Although there was a range of cancers included, 21 studies used exclusively female breast cancer samples, with one study using ovarian cancer [42], one gynaecological cancer [34] and two inclusive of breast cancer, but with the addition of others [6,41]. Owing to the low $n$ for cancers other than breast, an analysis of the moderating effects of cancer type could not be conducted. Therefore, a separate analysis was conducted on breast cancer alone, as this is the most frequently studied cancer population. Also, the moderating effects of gender were not analysed owing to the predominance of women over men in the final dataset.

\section{CR-PTSD prevalence rates}

The results of the meta-analysis are available in Table 2 . The table presents prevalence statistics for the whole pool of cancer studies and for breast cancer alone, grouped by

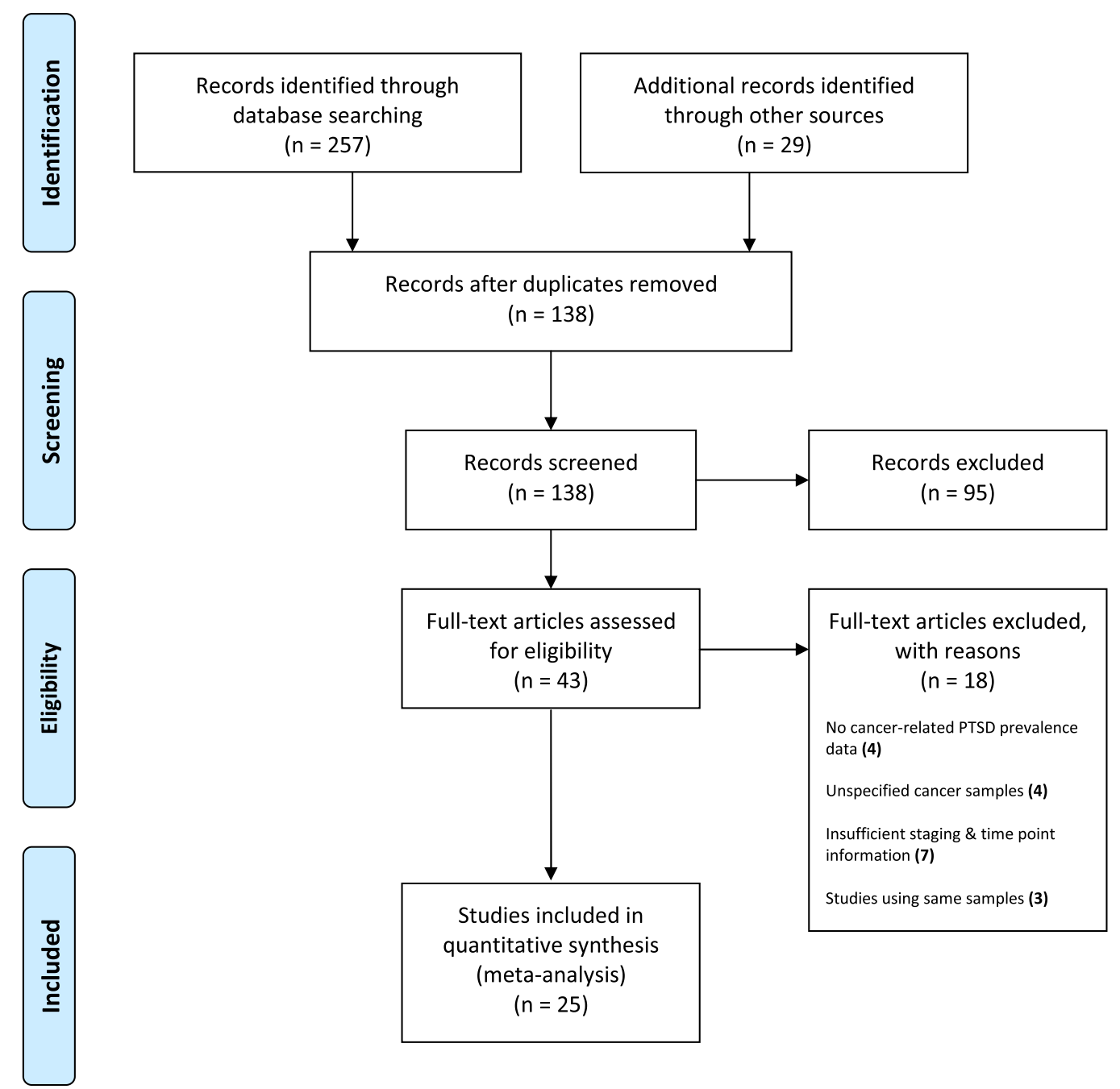

Figure I. PRISMA flow chart of systematic review procedure 
Table 2. PTSD event rates (a) across cancers and (b) for breast cancer samples, according to method of assessment

\begin{tabular}{|c|c|c|c|c|c|c|c|c|c|c|c|c|c|c|}
\hline \multirow{2}{*}{$\begin{array}{l}\text { Cancer-related PTSD } \\
\text { measure }\end{array}$} & & \multirow{2}{*}{$\begin{array}{l}\mathbf{k} \\
\%\end{array}$} & \multirow{2}{*}{$\begin{array}{l}\text { Event } \\
\text { rate }\end{array}$} & \multicolumn{2}{|c|}{$95 \% \mathrm{Cl}$} & \multirow[b]{2}{*}{$p$} & \multicolumn{4}{|c|}{ Heterogeneity } & \multicolumn{4}{|c|}{$\mathrm{Tau}^{2}$} \\
\hline & & & & Lower & Upper & & Q & d.f. & $I^{2}$ & $p$ & $\mathrm{Tau}^{2}$ & Tau & SE & Variance \\
\hline \multicolumn{15}{|l|}{ Breast cancer } \\
\hline \multicolumn{15}{|l|}{ By assessment method } \\
\hline \multirow[t]{2}{*}{ Interview } & Current & 10 & 5.8 & 3.3 & 10 & $<0.01$ & 33.4 & 9 & 73.05 & $<0.01$ & 0.61 & 0.78 & 0.42 & 0.18 \\
\hline & Lifetime & 6 & 11.5 & 6.3 & 20.1 & $<0.01$ & 27.01 & 5 & 81.5 & $<0.01$ & 0.53 & 0.73 & 0.44 & 0.19 \\
\hline \multirow[t]{2}{*}{ Questionnaire } & Cut-off & 9 & 6.4 & 4.2 & 9.7 & $<0.00 \mid$ & 17.56 & 8 & 54.44 & $<0.01$ & 0.24 & 0.49 & 0.23 & 0.06 \\
\hline & Cluster & 10 & 12.1 & 9.3 & 15.7 & $<0.01$ & 28.14 & 9 & 68.02 & $<0.01$ & 0.14 & 0.37 & 0.12 & 0.01 \\
\hline \multicolumn{15}{|l|}{ By specific tool } \\
\hline \multirow[t]{2}{*}{$\mathrm{SCID}$} & Current & 7 & 4.1 & 2 & 8.5 & $<0.00 \mid$ & 21.89 & 6 & 72.59 & $<0.001$ & 0.7 & 0.83 & 0.61 & 0.37 \\
\hline & Lifetime & 4 & 14.2 & 7.7 & 24.9 & $<0.00 \mid$ & 15.55 & 3 & 80.71 & $<0.00$ I & 0.4 & 0.63 & 0.42 & 0.17 \\
\hline \multirow[t]{2}{*}{ PCL-C } & Cut-off & 9 & 6.4 & 4.2 & 9.7 & $<0.001$ & 17.56 & 8 & 54.44 & $<0.03$ & 0.24 & 0.49 & 0.23 & 0.06 \\
\hline & Cluster & 9 & 11.2 & 8.7 & 14.4 & $<0.00 \mid$ & 18.8 & 8 & 57.45 & $<0.02$ & 0.1 & 0.31 & 0.1 & 0.01 \\
\hline \multicolumn{15}{|l|}{ Across cancers } \\
\hline \multicolumn{15}{|l|}{ By assessment method } \\
\hline \multirow[t]{2}{*}{ Interview } & Current & 12 & 6.4 & 4.1 & 9.9 & $<0.01$ & 42.8 & 11 & 74.29 & $<0.01$ & 0.41 & 0.64 & 0.34 & 0.11 \\
\hline & Lifetime & 7 & 12.6 & 7.4 & 20.7 & $<0.01$ & 28.73 & 6 & 79.2 & $<0.01$ & 0.49 & 0.7 & 0.38 & 0.15 \\
\hline \multirow[t]{2}{*}{ Questionnaire } & Cut-off & 10 & 7.3 & 4.5 & 11.3 & $<0.01$ & 31.4 & 9 & 71.33 & $<0.01$ & 0.47 & 0.68 & 0.33 & 0.11 \\
\hline & Cluster & 11 & 13.8 & 9.5 & 19.6 & $<0.01$ & 75.89 & 10 & 86.82 & $<0.01$ & 0.41 & 0.64 & 0.29 & 0.08 \\
\hline \multicolumn{15}{|l|}{ By specific tool } \\
\hline \multirow[t]{2}{*}{ SCID } & Current & 9 & 5.1 & 2.8 & 8.9 & $<0.001$ & 35.46 & 8 & 77.43 & $<0.001$ & 0.53 & 0.72 & 0.49 & 0.24 \\
\hline & Lifetime & 5 & 15.3 & 9.1 & 24.7 & $<0.00 \mid$ & 16.64 & 4 & 75.96 & $<0.00$ I & 0.34 & 0.59 & 0.33 & 0.11 \\
\hline \multirow[t]{2}{*}{ PCL-C } & Cut-off & 10 & 7.3 & 4.5 & 11.7 & $<0.00 \mid$ & 31.4 & 9 & 71.33 & $<0.001$ & 0.47 & 0.68 & 0.33 & 0.11 \\
\hline & Cluster & 9 & 11.2 & 8.7 & 14.4 & $<0.001$ & 18.8 & 8 & 57.45 & $<0.001$ & 0.09 & 0.31 & 0.01 & 0.01 \\
\hline
\end{tabular}

assessment strategy, from clinical interview methods (SCID, CAPS and Watson's PTSD Inventory) to questionnaires (PCL-C, PTSD Diagnostic Scale (PDS), PTSD Reaction Index and PTSD Scale). Separate statistics are provided for the PCL-C and SCID given their use in the majority of studies. See Supporting Information Figures 2-6 for forest and funnel plots.

All cancer types: Studies that used the PCL-C yielded lower event rates using the cut-off method $(7.3 \%, 95 \%$ $\mathrm{CI}=4.5-11.7, k=10)$ compared with the symptom cluster method $(11.2 \%, 95 \% \mathrm{CI}=8.7-14.4, k=9)$. Studies using the SCID alone yielded higher lifetime diagnoses $(15.3 \%, 95 \% \mathrm{CI}=9.1-24.7, k=5)$ than current diagnoses $(5.1 \%, 95 \% \mathrm{CI}=2.8-8.9, k=9)$. Studies using a clinical interview method yielded a combined event rate of $6.4 \%$ (95\% CI $=4.1-9.9, \quad k=12$ ) for current CR-PTSD and $12.6 \%(95 \% \mathrm{CI}=7.5-20.7, k=7)$ for lifetime CR-PTSD. Studies that used a cut-off score screening method used the PCL-C exclusively. All other screening tools used a symptom cluster method and yielded similar prevalence estimates to the PCL-C cluster method $(13.8 \%, 95 \% \mathrm{CI}=9.5-19.6$, $k=11$ ). No comparison could be performed between interview methods, or between screening tools, because only the PCL-C and the SCID were used more than once. Although the point estimates for each assessment method are comparable, each is characterised by considerable between-study heterogeneity across cancer samples (Table 2; $I^{2}=54-86 \%$ ).

Breast cancer: Studies that used the PCL-C yielded lower event rates using the cut-off method $(6.4 \%, 95 \%$
$\mathrm{CI}=4.2-9.7, k=9)$ compared with the symptom cluster method $(11.2 \%, 95 \% \mathrm{CI}=8.7-14.4, k=9)$. These figures are similar to those found across cancers, although the cut-off event rate for breast cancer alone is $1 \%$ less. The rates for the symptom cluster method here are the same for breast cancer as for all cancers. Studies using the SCID alone yielded lower but nevertheless comparable event rates for current CR-PTSD (4.1\%, 95\% $\mathrm{CI}=2-8.5, k=7)$. Much higher rates were found for lifetime CR-PTSD $(14.2 \%, 95 \% \mathrm{CI}=7.7-24.9, k=4)$. Studies that used a clinical interview method yielded a combined event rate of $5.8 \%(95 \% \mathrm{CI}=3.3-10, k=10)$ for current CR-PTSD and $11.5 \%(95 \% \mathrm{CI}=6.3-20.1, k=6)$ for lifetime CR-PTSD. Studies using the cut-off scoring method used the PCL-C exclusively (see aforementioned results). All other screening tools used a symptom cluster method and yielded remarkable similar prevalence estimates to the PCL-C cluster method $(12.1 \%, 95 \% \mathrm{CI}=9.3-15.7$, $k=10)$. Similar between-study heterogeneity was observed for breast cancer samples $\left(I^{2}=54.4-81.5 \%\right)$.

\section{Sources of heterogeneity}

Mean sample age, mean time since diagnosis and mean time since end of treatment were entered into a metaregression for their variance contribution to CR-PTSD prevalence rates. Early or advanced modal disease staging and population of origin (USA, UK and Europe, Japan and Israel) were analysed categorically to assess group differences in prevalence. 
Time post-diagnosis was not significant for any analyses. Time post-treatment was not significant when using the SCID, PCL-C or the questionnaire method but was significant when including other clinical interviews to assess lifetime CR-PTSD $\left[Q_{\text {model }}(1)=3.84, T_{\text {unexplained }}^{2}=0.22 k=3\right.$, $p=0.05]$, with CR-PTSD event rates decreasing when time since treatment increases, and a variance contribution of $56 \%\left(R^{2}=0.56, p<0.05\right)$. The contribution of mean sample age to prevalence was not significant for breast cancer samples, or when using the cut-off and cluster screening methods and the clinical interview method, or when using the SCID to assess lifetime CR-PTSD in all cancers. However, it was significant when using the SCID to assess current CR-PTSD $\left[Q_{\text {model }}(1)=0.43, p=0.05\right]$.

Disease stage was significant when using the SCID to assess current CR-PTSD [ $Q(1)=10.23, p=0.05]$. Studies characterised by early stages (I-II) yielded markedly lower prevalence rates $(4.2 \%, 95 \% \mathrm{CI}=2.1-8.1, k=8$, $n=737$ ) than those with advanced-stage (III-IV) disease $(11.4 \%, 95 \% \mathrm{CI}=9.6-13.5, k=1, n=989)$. This was also true for the interview method $[Q(1)=6.07, p=0.01]$, with the only difference in effect coming from the additional interview methods other than the SCID in the early-stage group $(5.7 \%, 95 \% \mathrm{CI}=3.4-9.6, k=11, n=970)$. Finally, the use of the questionnaire method with cluster scoring also yielded a significant difference $[Q(1)=3.71$, $p=0.05]$ with early stages yielding significantly lower prevalence rates $(11.6,95 \% \mathrm{CI}=8.8-13.5, k=9, n=1894)$ than advanced-stage samples $(31.395 \% \mathrm{CI}=11.8-61$, $k=2, n=112$ ). This moderator was not significant for pure breast cancer samples.

Population was not a significant moderator when using the questionnaire method or on the PCL-C, or the SCID, alone, and in either the full cancer sample or breast cancer samples. But significant differences were found when using the clinical interview method to diagnose current CR-PTSD $[Q(3)=9.2, \quad p=0.03]$, with Israel having significantly higher rates $(18 \%, 95 \% \mathrm{CI}=8.8-33.2$, $k=1)$ than the USA $(5.6 \%, 95 \% \mathrm{CI}=2.6-11.6, k=8)$, Europe $(6 \%, 95 \% \mathrm{CI}=2.4-14, k=3)$ or Japan $(3.9 \%$, 95\% CI $=1.8-8.4, k=1)$. Population was also significant for lifetime CR-PTSD $[Q(2)=11.28, p=0.03]$, with the USA $(15.4 \%, 95 \% \quad \mathrm{CI}=8.4-26.7, k=5)$ and Japan $(14.8 \%, 95 \% \mathrm{CI}=10-21.3, k=1)$ having higher rates than Europe $(2.8 \%, 95 \% \mathrm{CI}=1.1-7.2, k=1)$. Population was significant in exclusively breast cancer samples when diagnosing lifetime CR-PTSD with the interview method $[Q(2)=10.83, p<0.01]$, with the UK and Europe $(2.8 \%$, $95 \% \mathrm{CI}=1.1-7.2$ ) having a significantly lower prevalence than the USA $(14.2 \%, 95 \% \mathrm{CI}=6.8-27.5, k=4)$ and Japan $(14.8,95 \% \mathrm{CI}=10-21.3, k=1)$. Similar trends in breast cancer were found when diagnosing current CRPTSD $[Q(3)=11.43, p<0.01]$, with Israel yielding higher rates $(18 \%, 95 \% \mathrm{CI}=8.8-33.2, k=1)$ than Japan $(3.9 \%$,
$95 \% \mathrm{CI}=1.8-8.4, k=1), \mathrm{UK}$ and Europe $(4.1 \%, 95 \% \mathrm{CI}=$ $2.2-7.6, k=2)$ and $\mathrm{USA}(5.7 \%, 95 \% \mathrm{CI}=2.5-12.6, k=6)$.

\section{Discussion}

This is thought to be the first meta-analysis to investigate the prevalence of CR-PTSD in cancer survivors, and the contribution of between-study heterogeneity to the fluctuation of these rates. Our data for all cancers indicated that studies using clinical interview methods yield a mean prevalence of $6.4 \%$ for current CR-PTSD and $12.6 \%$ for lifetime CR-PTSD. Screening methods that used cluster scoring indicated a prevalence of $13.8 \%$ for clinically significant CR-PTSD symptoms. The PCL-C yielded lower event rates of clinically significant CR-PTSD symptoms using the cut-off $(7.3 \%)$ compared with symptom cluster $(11.2 \%)$, whereas the SCID yielded predictably higher lifetime diagnoses (15.3\%) than current diagnoses (5.1\%) for CR-PTSD.

These rates are similar to those found by Alter et al. [6] in the DSM-IV Field Trials (4\%), Green et al. [7] and Palmer et al. [35], which identify a current CR-PTSD prevalence of $2.5-5 \%$. Epidemiological surveys reveal the lifetime conditional risk (CR) of PTSD for women in the US general population to be between $10.4 \%(95 \% \mathrm{CI}=$ 8.8-11.7\%) [43] and $13 \%(95 \% \mathrm{CI}=9.9-16.1 \%)$ [44]. Nevertheless, the lifetime CR-PTSD rate for our $100 \%$ female US sample was $15.4 \%$ (95\% CI $=8.4-26.7 \%$ ), which is higher than the CR for the USA, but within the CIs for Breslau's survey [44]. If 5-12\% of cancer survivors develop CR-PTSD, a trauma framework may not represent the distress experienced by most patientsas argued by Green et al. [7], Palmer et al. [35] and now the DSM-5 task force [9]. This supports Kangas's criticism that the lack of differential diagnoses in semistructured clinical interviews challenges the validity of PTSD diagnosis [10]. It does not, however, take into account that the literature fails to record the presence/absence of discrete catastrophic events within the course of the disease that would make the cancer experience traumatic.

We recommend that clinicians consider the full range of presentations, be aware of the nature of cancer as a stressor and assess for discrete events that occurred during the treatment to warrant a PTSD diagnosis and, in the absence of these, consider a diagnosis of $\mathrm{AD}$. If cancer survivors meet $\mathrm{AD}$ criteria, this does not discount the possibility of PTSD development. The literature shows that cancer survivors present with subsyndromal symptoms $[7,45,46]$, which are also associated with considerable functional impairments [26,45]. Although this too may lead clinicians to initially question the validity of a CR-PTSD diagnosis, it does not discount the possibility that these subsyndromal symptoms may be a predictor of future PTSD. DSM-5 course specifiers state that PTSD may present with delayed expression, where only 
subsyndromal criteria are met before eventually meeting full criteria at least 6 months post-trauma [9]. This is supported by recent meta-analyses that show that delayed expression is preceded by high stress sensitivity, additional life stressors, maladaptation to continued exposure to stress and the steady accumulation of subsyndromal symptoms from the onset of the stressor $[47,48]$. These are features of cancer, and subsyndromal symptoms are also present at the beginning of cancer treatment, fluctuate and persist throughout the course of the disease and may, too, reflect a delayed PTSD presentation [15]. Although rightly considered an adjustment response during treatment, this may develop into CR-PTSD if those factors prevent the cognitive processing of the illness.

In our moderator analysis, screening questionnaires yielded higher rates of CR-PTSD symptoms than did the use of clinical interviews for current CR-PTSD. The reasons for this may be that whereas interviews diagnose disorder, questionnaires detect clinically significant symptoms, are not clinician administered and do not screen for disorders that better explain the symptoms [20]. However, the PCL$\mathrm{C}$ has demonstrated its reliable sensitivity and specificity in correctly identifying clinical cases [11], but there is still debate on where to place the cut-off score [20]. The Davidson Trauma Scale [49] can be used as an alternative to screen for CR-PTSD. It has a cut-off score that moves depending on the prevalence of PTSD in the specific population. As this meta-analysis revealed a current CRPTSD prevalence of $6.4 \%$, the Davidson cut-off for the cancer survivor population would be 47 .

When lifetime CR-PTSD was assessed using the SCID, prevalence decreased with time post-treatment. This finding should be interpreted with caution. Firstly, this trend was only observed for lifetime CR-PTSD, and secondly, this factor is a proxy variable for time post-trauma. As there is no agreement as to whether the diagnosis or the treatments are the definitive traumas, investigators adopted one of the two indices; therefore, these moderators could not be combined, resulting in low statistical power. However, this finding reflects the epidemiologic trend that non-cancer PTSD sufferers do recover naturally over time, although a minority of them remain symptomatic after many years [43]. This has implications for the survivors, as potential cases may not be identified during routine follow-up, placing them at risk of PTSD months to years after their treatment.

Younger-aged samples were associated with higher current CR-PTSD event rates on the SCID. This trend was just significant, so these results too must be interpreted cautiously. The majority of research into this relationship concentrates on age at diagnosis-our study-level moderator was the mean age of the sample. Several of the samples were many months, or sometimes years, post-diagnosis, and this likely reduced the sensitivity of our analysis - but as it addressed the impact of younger age on the prevalence rates of lifetime CR-PTSD (not on symptom severity) - it provides a singular contribution.

The final aim was to synthesise disease-stage data to establish whether they are a risk factor for PTSD following comments that skewed samples characterised the research [1]. Our post-hoc analyses suggested that advanced disease is related to an increase in CR-PTSD event rates on PCL-C and on the SCID. This is commensurate with studies that show a positive relationship between disease severity and CR-PTSD $[8,27,33,34]$ and may provide some preliminary support for this conclusion, but we advise cautious interpretation. Although the differences in event rates were significant, additional factors may have contributed. The advanced disease sample in one study [41] was from a different culture and set in palliative care, which introduces environmental factors into the development of CR-PTSD that are uncharacteristic of early-stage cancer samples. The degrees of freedom for the early-stage group were larger than those for the advanced-stage group and reflect the abundance of earlier stage cancers in the majority of studies.

\section{Publication bias}

The funnel plots revealed substantial biases. However, this is not the bias one would expect from the file-drawer problem. Publication bias is identified by a skew in the distribution towards higher effect sizes, at the bottom of the funnel plot. The presence of this type of bias is not visually depicted in our plots.

\section{Strengths and limitations}

There are limitations that constrain our conclusions. The findings from the moderator analysis are tentative because several findings come from retaining $k=1$ comparisons. This is problematic as the $Q$ test has low power to detect heterogeneity when $k$ is low [50]. Nevertheless, this highlights the need for concise, standardised and transparent reporting to facilitate future meta-analytic studies. Also, only study-level moderators were included. Many studies did not assess psychiatric history or additional life stress at the time of assessment, so intrapersonal vulnerability factors were unaccounted for. The substantial variability in reporting styles, and limitations of using CMA, may have introduced variance into analyses that is not attributable to the moderators of interest. Studies that have used questionnaires may over-inflate rates of CR-PTSD owing to symptom endorsement being confounded by artefacts of cancer drugs, and medical conditions [5], and realistic fears of cancer recurrence being endorsed as the acceptance of a foreshortened future. Nevertheless, the sensitivity and specificity of the PCL-C against the SCID for CR-PTSD are sufficient to screen for those survivors who may be suffering from an $\mathrm{AD}$ or CR-PTSD. Out of the studies that have used interviews, few have assessed the rates of co-morbid 
disorders, meaning CR-PTSD might not be the primary disorder. Also, all of the studies included in this metaanalysis used DSM-IV criteria, not the new DSM-5. Early epidemiological studies documented the inflation of PTSD prevalence owing to the revised DSM-IV criteria, so PTSD prevalence may decrease owing to the new DSM-5 PTSD criterion A. The strengths of this investigation are that it included 25 studies and a substantial patient pool across several populations. This affords our analysis generalisability that the individual studies could not achieve [35]. Precision was enhanced by including data for symptoms anchored to the experience of adult cancer. This does not account for differential diagnoses but does account for stress-related symptoms due to an extreme stressor. In this case, the findings may reflect the proportion of survivors who meet criteria for AD and PTSD. This is more clinically useful than focusing on PTSD alone.

\section{Future research}

If the cancer experience is not generally traumatic, then the fact that CR-PTSD does emerge in a significant minority of survivors suggests that other factors affect adjustment and the cognitive processing of the illness. It has been argued that 'less traumatic' stressors can be expected to trigger PTSD in vulnerable individuals who have limited resources to manage their response to stress [51] and that the insufficiency of some events to meet PTSD criterion A may reflect failure to consider the role of personal stress reactivity [52,53], and the effect of allostatic load [53]. As such, there is less information on variables that influence this adjustment. Variables such as social support and/or constraints on talking about the illness [21,28,30,54-58], emotion regulation strategies such as dissociation [22], emotional suppression and avoidance [59,60], and rumination [61] have been identified as predictors of PTSD symptoms in cancer populations. Most recently, emotional processing styles have been implicated in post-natal depression [62] and PTSD $[63,64]$. Given the protracted nature of cancer, it is possible that social variables and their relationship to beliefs about experiencing and expressing of emotions may influence cognitive processing over the course of disease and predict the emergence of CR-PTSD. This necessitates further investigation on the role of these variables in multiple oncology services both nationally and internationally. But firstly, in order to assess the risk of CR-PTSD in this population, it may be necessary to report, or make readily available, the raw data on CR-PTSD event rates by (a) disease stage, (b) cancer type, (c) treatment regimens, (d) any traumatic events during the course of the disease, (e) age at diagnosis and (f) the mean time of assessment in relation to time post-diagnosis and time post-treatment.

\section{Conclusions}

Prevalence rates from questionnaires reveal that a minority of cancer survivors present with clinically significant symptoms due to cancer. In these cases, we recommend that oncology specialists recognise the possibility of CR-PTSD and refer their patient to mental health services. Prevalence rates from clinical interviews show that a minority of cancer survivors meet (or have previously met) full DSM-IV criteria for CR-PTSD after the conclusion of treatment and that those who are younger, diagnosed with more advanced disease or recently completed treatment may be at greater risk of CR-PTSD. Methodological heterogeneity prohibits robust conclusions about the expected prevalence of CRPTSD as a primary disorder but does provide some clinical justification for the diagnosis of a CR-PTSD in a minority of cases. Given the release of the DSM-5, we recommend caution in diagnosing CR-PTSD but advise further investigation into whether traumatic occurrences were experienced during the course of the disease.

\section{Acknowledgements}

The primary author would like to thank Katharina Ingund Brüning for her independent rater work on the meta-analysis dataset. This study was match funded by Bournemouth University and The Royal Bournemouth and Christchurch Hospitals NHS Foundation Trust as part of a series of studies towards the award of $\mathrm{PhD}$ for the first author.

\section{Conflict of interest}

The authors have declared no conflicts of interest.

\section{References}

1. Gurevich M, Devins GM, Rodin GM. Stress response syndromes and cancer: conceptual and assessment issues. Psychosomatics 2002;43:259-281, doi:10.1176/appi.psy.43.4.259.

2. Kangas M, Henry JL, Bryant RA. Posttraumatic stress disorder following cancer. A conceptual and empirical review. Clin Psychol Rev 2002;22:499-524, doi:10.1016/S02727358(01)00118-0.

3. Koutrouli N, Anagnostopoulos F, Potamianos G. Posttraumatic stress disorder and posttraumatic growth in breast cancer patients: a systematic review. Women Health 2012;52:503-516, doi:10.1080/03630242.2012.679337.

4. Smith MY, Redd WH, Peyser C, Vogl D. Post-traumatic stress disorder in cancer: a review. Psycho-Oncology 1999;8:521-537, doi:10.1002/(SICI)1099-1611(199911/12) 8:6<521::AID-PON423>3.0.CO;2-X.

5. American Psychiatric Association. Diagnostic and Statistical Manual of Mental Disorders (4th edn). Author: Washington, DC, 1994.

6. Alter CL, Pelcovitz D, Axelrod A, et al. Identification of PTSD in cancer survivors.
Psychosomatics 1996;37:137-143, doi:10.1016/ S0033-3182(96)71580-3.

7. Green BL, Rowland JH, Krupnick JL, et al. Prevalence of posttraumatic stress disorder in women with breast cancer. Psychosomatics 1998;39:102-111, doi:10.1016/S0033-3182 (98)71356-8.

8. Mundy EA, Blanchard EB, Cirenza E, Gargiulo J, Maloy B, Blanchard CG. Posttraumatic stress disorder in breast cancer patients following autologous bone marrow transplantation or conventional cancer treatments. Behav Res Ther 2000;38:1015-1027, doi:10.1016/ S0005-7967(99)00144-8. 
9. American Psychiatric Association. Diagnostic and Statistical Manual of Mental Disorders (5th edn). Author: Washington, DC, 2013.

10. Kangas M. DSM-5 trauma and stress-related disorders: implications for screening for cancer-related stress. Front Psychiat 2013, doi:10.3389/fpsyt.2013.00122.

11. Andrykowski MA, Cordova MJ, Studts JL, Miller TW. Posttraumatic stress disorder after treatment for breast cancer: prevalence of diagnosis and use of the PTSD Checklist-Civilian Version (PCL-C) as a screening instrument. $J$ Consult Clin Psychol 1998;66:586-590,s doi:10.1037/0022-006X.66.3.586.

12. Blake DD, Weathers FW, Nagy LM, et al. The development of a clinician-administered PTSD scale. J Trauma Stress 1995;8:75-90, doi:10.1002/jts.2490080106.

13. First MB, Spitzer RL, Gibbon M, Williams JBW. Structured Clinical Interview for DSM-IV-TR Axis I Disorders, Research Version, Non-patient Edition. (SCID-I/NP) New York: Biometrics Research, New York State Psychiatric Institute, 2002.

14. Blanchard EB, Jones-Alexander J, Bucklet TC, Forneris CA. Psychometric properties of the PTSD Checklist (PCL). Behav Res Ther 1996;34:669-673, doi:10.1016/0005-7967 (96)00033-2.

15. Andrykowski MA, Cordova MJ, McGrath PC, Sloan DA, Kenady DE. Stability and change in posttraumatic stress disorder symptoms following breast cancer treatment: a 1-year follow-up. Psycho-Oncology 2000;9:69-78, doi:10.1002/(SICI)1099-1611 (200001/02)9:1<69::AID-PON439>3.0. $\mathrm{CO} ; 2-\mathrm{R}$.

16. Andrykowski MA, Cordova MJ, Studts JL, Miller TW. Posttraumatic stress disorder after treatment for breast cancer: prevalence of diagnosis and use of the PTSD Checklist-Civilian Version (PCL-C) as a screening instrument. $J$ Consult Clin Psychol 1998;66:586-590, doi:10.1037/0022-006X.66.3.586.

17. Cordova MJ, Studts JL, Hann DM, Jacobsen PB, Andrykowski MA. Symptom structure of PTSD following breast cancer. $J$ Trauma Stress 2000;13:301-319, doi:10.1023/A:1007762812848.

18. Smith MY, Redd W, DuHamel K, Vickberg SJ, Ricketts P. Validation of the PTSD Checklist- Civilian Version in survivors of bone marrow transplantation. J Trauma Stress 1999;12:485-499, doi:10.1023/A:1024719104351.

19. Shelby RA, Golden-Kreutz DM, Andersen BL. Mismatch of posttraumatic stress disorder (PTSD) symptoms and DSM-IV symptom clusters in a cancer sample: exploratory factor analysis of the PTSD Checklist-Civilian Version. J Trauma Stress 2005;18:347-357, doi: $10.1002 /$ jts. 20033 .

20. Thompson SB, Eccleston L, Hickish T. Posttraumatic stress disorder in cancer survivors: recognising and acknowledging the symptoms. Webmed Cent Oncol 2011;2:WMC002062
21. Kornblith AB, Herndon JE, Weiss RB, et al. Long-term adjustment of survivors of early-stage breast carcinoma, 20 years after adjuvant chemotherapy. Cancer 2003;98:679-689, doi:10.1002/ cncr.11531.

22. Kangas M, Henry JL, Bryant RA. Predictors of posttraumatic stress disorder following cancer. Health Psychol 2005a;24:579-585, doi:10.1037/ 0278-6133.24.6.579.

23. Levine EG, Eckhardt J, Targ E. Change in posttraumatic stress symptoms following psychosocial treatment for breast cancer. Psycho-Oncology 2005;14:618-635, doi:10.1002/pon.882.

24. Morrill EF, Brewer NT, O'Neill SC, et al. The interaction of post-traumatic growth and posttraumatic stress symptoms in predicting depressive symptoms and quality of life. Psycho-Oncology 2008;17:948-953, doi:10.1002/pon.1313.

25. Pitman RK, Lanes DA, Williston SK, et al. Psychophysiologic assessment of posttraumatic stress disorder in breast cancer patients. Psychosomatics 2001;42:133-140, doi:10.1176/appi.psy.42.2.133.

26. Cordova MJ, Andrykowski MA, Kenady DE, McGrath PC, Sloan DA, Redd WH. Frequency and correlates of posttraumatic-stress-disorder-like symptoms after treatment for breast cancer. J Consult Clin Psychol 1995;63:981-986, doi:10.1037/ 0022-006X.63.6.981.

27. Jacobsen PB, Widows MR, Hann DM, Andrykowski MA, Kronish LE, Fields KK. Posttraumatic stress disorder symptoms after bone marrow transplantation for breast cancer. Psychosom Med 1998;60:366-371, doi:10.1097/00006842-199805000-00026.

28. Cordova MJ, Giese-Davis J, Golant M, Kronenwetter C, Chang V, Spiegel D. Breast cancer as trauma: posttraumatic stress and postraumatic growth. J Clin Psychol Med Settings 2007;14:308-319, doi:10.1007/s10880-007-9083-6.

29. Epping-Jordan JE, Compas BE, Osowiecki $\mathrm{DM}$, et al. Psychological adjustment in breast cancer: processes of emotional distress. Health Psychol, 1999;18:315-326, doi:10.1037/0278-6133.18.4.315.

30. Green BL, Krupnick JL, Rowland JH, et al. Trauma history as a predictor of psychologic symptoms in women with breast cancer. $J$ Clin Oncol 2000;18:1084-1093.

31. Koopman C, Butler LD, Classen C, et al. Traumatic stress symptoms among women with recently diagnosed primary breast cancer. J Trauma Stress 2002;15:277-287, doi:10.1023/A:1016295610660.

32. Edmondson D, Richardson S, Falzon L, Davidson KW, Ali M. Posttraumatic stress disorder prevalence and risk of recurrence in acute coronary syndrome patients: a metaanalytic review. PLoS One 2012;7:e38915, doi:10.1371/journal.pone.0038915.

33. Boyer BA, Bubel D, Jacobs SR, et al. Posttraumatic stress in women with breast cancer and their daughters. Am J Fam Ther 2002;30:323-338, doi:10.1080/01926180290 033466 .
34. Posluszny DM, Edwards RP, Dew MA, Baum A. Perceived threat and PTSD symptoms in women undergoing surgery for gynaecologic cancer or benign conditions. Psycho-Oncology 2011;20:783-787, doi:10.1002/ pon. 1771.

35. Palmer SC, Kagee A, Coyne JC, DeMichele A. Experience of trauma, distress, and posttraumatic stress disorder among breast cancer patients. Psychosom Med 2004;66:258-264, doi:10.1097/01. psy.0000116755.71033.10.

36. Watson CG, Juba MP, Manifold V, Kucala T, Anderson PED. The PTSD interview: rationale, description, reliability, and concurrent validity of a DSM-III-based technique. J Clin Psychol 1991;47:179-188, doi:10.1002/10974679(199103)47:2<179::AID-JCLP2270470 202>3.0.CO;2-P.

37. Steinberg AM, Brymer MJ, Kim S, et al. Psychometric properties of the UCLA PTSD Reaction Index: part 1, J Trauma Stress, 2013;26:1-9, doi:10.1002/jts.21755.

38. Joseph S. Psychometric evaluation of Horowitz's Impact of Event Scale: a review. J Trauma Stress 2000;13:101-113, doi:10.1023/A:1007777032063.

39. Comprehensive Meta-analysis (version 2) [computer software]. 2005; Englewood, NJ: Biostat.

40. Moher D, Liberati A, Tetzlaff J, Altman DG. The PRISMA Group. Preferred reporting items for systematic reviews and meta-analyses: the PRISMA statement. BMJ 2009;339:b2535, doi:10.1136/bmj.b2535.

41. Mystakidou K, Parpa E, Tsilika E, et al. Traumatic experiences of patients with advanced cancer. J Loss Trauma 2012;17:125-136, doi:10.1080/15325024.2011.595296.

42. Goncalves V, Jayson G, Tarrier N. A longitudinal investigation of posttraumatic stress disorder in patients with ovarian cancer. J Psychosom Res 2011;70:422-431, doi:10. 1016/j.jpsychores.2010.09.017.

43. Kessler RC, Sonneha A, Bromet E, Hughes $\mathrm{M}$, Nelson CB. Posttraumatic stress disorder in the National Co-morbidity Survey. Arch Gen Psychiatry 1995;52:1048-1060, doi:10.1001/ archpsyc. 1995.03950240066012.

44. Breslau N, Kessler RC, Chilcoat HD, Schultz LR, Davis GC, Andreski P. Trauma and posttraumatic stress disorder in the community: the 1996 Detroit Area Survey of Trauma. Arch Gen Psychiatry 1998;55:626-632, doi:10.1001/ archpsyc. 1995.03950240066012.

45. Shelby RA, Golden-Kreutz DM, Andersen BL. PTSD diagnoses, subsyndromal symptoms, and co morbidities contributing to impairments for breast cancer survivors. $J$ Trauma Stress 2008;21:165-172, doi:10.1002/jts.20316.

46. Guglietti CL, Rosen B, Murphy KJ, et al. Prevalence and predictors of posttraumatic stress in women undergoing an ovarian cancer investigation. Psychological Serv 2010;7:266-274, doi:10.1037/a0020338. 
47. Andrews B, Brewin, CR, Stewart L, Philpott R, Hejdenberg J. Comparison of immediate-onset and delayed-onset posttraumatic stress disorder in military veterans. $J$ Abnorm Psychol 2009;118:767-777, doi:10.1037/a0017203.

48. Smid GE, Mooren TT, van der Mast RC, Gersons BP, Kleber RJ. Delayed posttraumatic stress disorder: systematic review, meta-analysis, and metaregression analysis of prospective studies. J Clin Psychiatry 2009;70:1572Y1582, doi:10.4088/JCP.08r04484.

49. Davidson JRT, Book SW, Colket JT, Tupler LA. Assessment of a new selfrating scale for post-traumatic stress disorder. Psychol Med: J Res Psychiatr Allied Sci 1997;27:153-160.

50. Gavaghan DJ, Moore RA, McQuay HJ. An evaluation of homogeneity tests in metaanalyses in pain using simulations of individual patient data. J Int Assoc Study Pain 2000;85:415-424, doi:10.1016/S0304-3959 (99)00302-4.

51. Marshall RD, Amsel L, Suh EJ. Response to McNally and Breslau (2008). Am Psychol 2008;63:283-285.

52. King DW, King LA, Fairbank JA, Keane TM, Adams G. Resilience-recovery factors in post-traumatic stress disorder among female and male Vietnam veterans: hardiness, postwar social support, and additional stressful life events. J Pers Soc Psychol 1998;74:420-434, doi:10.1037/ 0022-3514.74.2.420.

53. King DW, King LA, Foy DW, Keane TM, Fairbank JA. Posttraumatic stress disorder in a national sample of female and male Vietnam veterans: risk factors, war-zone stressors, and resilience-recovery variables. $J$ Abnorm Psychol 1999;108:164-170, doi:10.1037/ 0021-843X.108.1.164.

54. Jacobsen PB, Sadler IJ, Booth-Jones M, Soety E, Weitzner MA, Fields KK.
Predictors of post-traumatic stress disorder symptomatology following bone marrow transplantation for cancer. $J$ Consult Clin Psychol 2002;70:235-240, doi:10.1037/0022006X.70.1.235.

55. Andrykowski MA, Cordova MJ. Factors associated with PTSD symptoms following treatment for breast cancer: test of the Andersen model. J Trauma Stress 1998;11:189-203, doi:10.1023/A:1024490718043.

56. Lepore SJ, Helgeson VS. Social constraints, intrusive thoughts, and mental health after prostate cancer. J Social Clin Psychol 1998;17: 89-106, doi:10.1521/jscp.1998.17.1.89.

57. Manne SL. Intrusive thoughts and psychological distress among cancer patients: the role of spouse avoidance and criticism. $J$ Consult Clin Psychol 1999;67:539-546, doi:10.1037//0022006X.67.4.539.

58. Widows MR, Jacobsen PB, Fields KK. Relation of psychological vulnerability factors to posttraumatic stress disorder symptomatology in bone marrow transplant recipients. Psychosom Med 2000;62:873-882, doi:10.1097/00006842-200011000-00018.

59. Amir M, Ramati A. Post-traumatic symptoms, emotional distress, and quality of life in long-term survivors of breast cancer: a preliminary research. $J$ Anxiety Disord 2002;16;191-206, doi:10.1016/ S0887-6185(02)00095-6.

60. Hampton MR, Frombach I. Women's experience of traumatic stress in cancer treatment. Health Care Women Int 2000;21:67-76, doi:10.1080/073993300245410.

61. Chan MWC, Ho SMY, Tedeschi RG, Leung CWL. The valence of attentional bias and cancer-related rumination in posttraumatic stress and posttraumatic growth among women with breast cancer. PsychoOncology 2011;20:544-552, doi:10.1002/ pon. 1761 .
62. Wilkins C, Baker R, Bick D. Thomas P. Emotional processing in childbirth: a predictor of postnatal depression? $\mathrm{Br} \quad \mathrm{J}$ Midwifery 2009;17:154-159, doi:http://dx.doi.org./10.1296 8/bjom.2009.17.3.40077

63. Baker R. Understanding Trauma: How to Overcome Post-traumatic Stress. Lion Hudson: Oxford, 2010.

64. Baker R, Gale L, Abbey G, Thomas S. Emotional processing therapy for posttraumatic stress disorder. Counsell Psychol Q 2013;26:362-385, doi:10.1080/09515070. 2013.816840.

65. Luecken LJ, Dausch B, Gulla V, Hong R, Compas BE. Alterations in morning cortisol with PTSD in women with breast cancer. $J$ Psychosom Res 2004;56:13-15, doi:10.1016/ S0022-3999(03)00561-0.

66. Matsuoka Y, Inagaki M, Sugawara Y, Imoto S, Akechi T, Uchitomi Y. Biomedical and psychosocial determinants of intrusive recollections in breast cancer survivors. Psychosomatics 2005;46:203-211, doi:10.1176/appi. psy.46.3.203.

67. Mehnert A, Koch U. Prevalence of acute and post-traumatic stress disorder and comorbid mental disorders in breast cancer patients during primary cancer care: a prospective study. Psycho-Oncology 2007;16:181-199, doi:10.1002/pon.1057.

68. Mehnert A, Koch, U. Psychological comorbidity and health-related quality of life and its association with awareness, utilization, and need for psychosocial support in a cancer register-based sample of longterm breast cancer survivors. $J$ Psychosom Res 2008;64:383-391, doi:10.1016/j. jpsychores.2007.12.005.

69. Gandubert C, Carriere I, Escot C, et al. Onset and relapse of psychiatric disorders following early breast cancer: a case-control study. Psycho-Oncology 2009;18:1029-1037, doi:10.10 02/pon.1469.

\section{Supporting information}

Additional supporting information may be found in the online version of this article at the publisher's web site. 